\title{
COLLABORATIVE PLANNING FOR THE ENVIRONMENTAL SUSTAINABILITY OF THE HAJJ
}

\author{
Abdullah Abonomi', Terry De Lacy ${ }^{2} \&$ Joanne Pyke ${ }^{3}$ \\ 1,2 Institute of Sustainable Industries and Livable Cities, Victoria University, \\ ${ }^{3}$ School for the Visitor Economy, Victoria University \\ (abdullah.abonomi@live.vu.edu.au, terry.delacy@vu.edu.au, joanne.pyke@vu.edu.au)
}

\begin{abstract}
Every year, millions of Muslim worshippers visit Mecca in Saudi Arabia to perform the Hajj which is the fifth and final pillar of Islam. Mecca hosts more than 2,300,000 people from around 183 different countries and cultures every year. In 2016, these numbers were forecast to grow to 2,500,000 in 2020 . This goal, however, has not been achieved due to the COVID-19 pandemic. The pandemic has forced the government to severely reduce the number of pilgrims in 2020 to just 10,000 people. Ultimately, this situation is temporary and visitor numbers should continue to rise. Tourism, especially religious tourism such as the Hajj, is expected to boost the economy and create new jobs for Saudi youth in the services sector. Yet, despite the many benefits of the pilgrimage, the Hajj itself has several severe adverse environmental impacts. The activities of Hajj generate considerable solid and liquid waste, use large quantities of scarce fresh water and produce high levels of greenhouse gasses (GHGs).
\end{abstract}

KEYWORDS: The Hajj, stakeholders' collaboration, environmental sustainability, greenhouse gasses

\section{PURPOSE AND BACKGROUND}

After launching Vision 2030, the government of Saudi Arabia has set many strategies and policies to reduce the significate impact of the Hajj activities on environmental sustainability. However, these environmental strategies have not been achieved due to a lack of stakeholder's collaboration.

For example, several hospitality agencies rejected requests for collaboration in preparing a meal for pilgrims that the government hoped would reduce waste. Furthermore, many hospitality agencies did not collaborate in implementing the Green Hajj project. Based on a wider study the success of sustainability plans and projects in a tourism context requires collaboration between stakeholders from all sectors (Graci, 2013; Jamal \& Getz, 1995), which does not exist presently in the Hajj.

Accordingly, the over-riding aim of this research is to investigate how the environmental sustainability of the Hajj can be improved. The particular focus of the study, however, is on how stakeholder collaboration can be improved to achieve sustainability goals. This study applies Gray's (1989) collaborative planning model to investigate the nature of the collaborative process between Hajj stakeholders and the drivers that induce the collaboration.

\section{METHODOLOGY}

To achieve the aim, this research employed a qualitative methodology with the objective of investigating the nature of collaboration between Hajj stakeholders while planning for environmental sustainability of the Hajj. Both primary data and secondary data was used in this study. 
A qualitative approach was adopted as the best strategy to understand why, as opposed to what things occur in the social world. The specific methods used in this study include unstructured and semi-structured interviews. The study was conducted in two rounds. Interviewees were selected to be representative of the public and private sector stakeholders and interviews conducted in a face-to-face setting at a convenient time and location. The qualitative analysis programme, Atlas, was used to analyse the data and define the themes of the research paper.

\section{FINDINGS}

Research findings show that there were many barriers to collaboration between Hajj stakeholders at each stage of Gray's collaboration model. Centralisation, leadership style, and lack of adequate resources in the collaboration process have added to the number of obstacles facing Hajj stakeholders to achieve environmental sustainability in Mecca.

Thus, since collaboration is vital to achieving environmental sustainability goals and objectives (Graci, 2013; Jamal \& Getz, 1995), decentralisation of the planning process, the presence of an effective leader, and existence of adequate resources is necessary to ensure the success of collaboration between Hajj stakeholders.

\section{CONCLUSION}

This study aims to investigate the nature of collaboration between Hajj stakeholders and the drivers that induce the collaboration at the planning stage. The ultimate aim of the investigation was to enhance the environmental sustainability of Hajj and the travel and stay in Mecca. This is because collaboration in the planning process has proven to be vital to achieving sustainability at tourist destinations (Graci, 2013).

The results show that the level of collaboration between stakeholders in Hajj is fragile at best and is still in the first phase. Despite the fact that on paper the project plans show that project planning has reached the third phase (implementation). In practical and realistic terms on the ground the level of collaboration between Hajj stakeholders remains in early stage.

Thus, this study aims to provide an analysis on how to improve the collaborative network between Hajj stakeholders when planning for and protecting the environment and ensuring sustainability of the Hajj.

\section{CONTRIBUTION/PRACTICAL IMPLICATIONS}

This paper aims to contribute to the existing theory by providing a different approach as to how collaboration can be achieved in developing countries that share a similar structural system to Saudi Arabia. The results demonstrate that a "top-down" approach can contribute positively to building a successful collaboration between tourism stakeholders. To illustrate, when the government included the environmental sustainability objectives in its Vision 2030 document the Hajj stakeholders started to collaborate with the aim of reducing the environmental impact of the Hajj.

Without the government intervention, this study argues that collaboration between Hajj stakeholders to set plans for preserving the environment and ensuring the sustainability of the Hajj pilgrimage would not have occurred. This contrasts with the already established argument that tourism destinations have executed a top-down approach in which the governmental sector sets the strategy and determines what 
is best for the destination is more likely to hinder the achievement of participatory and collaborative planning processes.

Moreover, the use of inductive methods has contributed in providing a better view of the main problems that have hampered the acceptable degree of stakeholder collaboration in the planning process, as well as the drivers that may enhance their level of collaboration. From a practical standpoint, the results of this study are beneficial for policymakers to understand the importance of changing their traditional approach in the planning process. Especially in this time as the government of Saudi Arabia is strongly attempting to improve the environmental sustainability as mentioned in the national document of Vision 2030.

Thus, collaboration between stakeholders from all sectors is one of the keys that need to exist to achieve this aim (Graci, 2013). The results may guide Hajj stakeholders from all sectors to understand the barriers and drivers that impact the development of collaboration. By doing this they may review and establish collaborative networks in the planning process.

\section{REFERENCES}

Graci, S. (2013). Collaboration and partnership development for sustainable tourism. Tourism Geographies, 15(1), 25-42.

Gray, B. (1989). Collaborating: Finding common ground for multiparty problems. San Francisco: JosseyBass.

Jamal, T. B., \& Getz, D. (1995). Collaboration theory and community tourism planning. Annals of Tourism Research, 22(1), 186-204. 